\title{
Evaluation of antioxidant and anti-lipid peroxidation potentials of Nigella sativa and onion extract on nicotine-induced lung damage
}

\author{
S.M. Zaki ${ }^{1,2}$ \\ ${ }^{1}$ Fakeeh College for Medical Sciences, Jeddah, Saudi Arabia \\ 2Department of Anatomy and Embryology, Faculty of Medicine, Cairo University, Cairo, Egypt \\ [Received: 17 November 2018; Accepted: 15 December 2018]
}

Background: The present work aimed to compare the protective effect of Nigella sativa (NS) and onion extract on the nicotine-induced lung damage in rats. The antioxidant and anti-lipid peroxidation potentials of both agents on nicotine-induced lung damage were studied.

Materials and methods: Thirty-six Sprague-Dawley albino rats, treated for 18 weeks, were divided into six groups: one negative control group, two positive control groups (oral onion and oral NS), nicotine-treated group, onion extract-treated group (concomitant nicotine and onion extract) and NS-treated group (concomitant nicotine and NS oil). The assessment of lung structure was based on haematoxylin and eosin and transmission electron microscopy. Lung malondialdehyde (MDA), superoxide dismutase activity (SOD), catalase (CAT), lung glutathione (GSH), and epithelial lining fluid GSH (ELF GSH) were used for assessment of the antioxidant and anti-lipid peroxidation potentials of NS and onion extract.

Results: The lung of the nicotine-treated group exhibited emphysematous air spaces, collapsed corrugated alveoli with ruptured interalveolar septa in some specimen and thickened septa in the others, massive congestion, extravasation of red blood cells, inflammatory cellular infiltration and fluid exudate. Much improvement was observed in the onion-treated group despite the presence of residual pathological affection. The lung in the NS-treated group showed the nearly normal architecture with slight congestion. Administration of nicotine promoted lipid peroxidation (elevation of MDA) and decreased the level of the antioxidant markers (SOD, CAT, lung GSH and ELF GSH). With the use of onion extract and NS, the level of MDA decreased by $17.85 \%$ and $35.71 \%$ while the levels of SOD, CAT, GSH, and ELF GSH increased. The increase was more prominent in the NS-treated group. The levels in the NS-treated group reached nearly the level markers of the control group.

Conclusions: Nigella sativa and onion extract attenuate the pathological effect of nicotine in the lung rats through antioxidative and anti-lipid peroxidative mechanisms with higher protection to NS. (Folia Morphol 2019; 78, 3: 554-563)

Key words: Nigella sativa, onion extract, nicotine, lung 


\section{INTRODUCTION}

Nicotine is a naturally occurring alkaloid found in a wide variety of plants [45]. Its chief source of contact is through the use of tobacco, nicotine-containing gum and nicotine replacement therapies such as transdermal nicotine patches [45]. Nicotine has dose-related harmful pulmonary effects that result in damage to the lung endothelial barrier, acute lung inflammation, and reduced lung endothelial cell proliferation [37]. Some researchers detected emphysematous changes with pulmonary congestion, oedema, and haemorrhage into alveoli and interstitial areas with the use of nicotine in rats [11]. In addition, nicotine causes lung cancer as well as obstructive pulmonary diseases [13].

The mechanism of nicotine by which it induces such damages is not completely delineated. Considerable evidence points to the involvement of oxidative stress [45]. Nicotine disrupts the antioxidant defence mechanisms by decreasing levels of glutathione (GSH) and superoxide dismutase (SOD) and by changing the balance between antioxidant capacity and oxidative stress-induced free radicals $[41,46]$. In addition, nicotine stimulates lipid peroxidation by increasing the malondialdehyde (MDA) and lactate dehydrogenase [46].

Nigella sativa (NS) is an annual flowering plant, native to Asia and the Middle East. The seed oil of NS is rich in polyphenols, tocopherols, protein, carbohydrates, vegetable oil and omega- 3 and omega- 6 fatty acids. The NS seed exhibits analgesic, antibacterial, anti-inflammatory, and antineoplastic activity [3]. In addition, NS oil was shown to have an antioxidant effect [18]. The anti-inflammatory effect of NS is comparable to $100 \mathrm{mg} / \mathrm{kg}$ acetylsalicylic acid [2]. The protective effects of NS oil in different tissues against toxic agents in animal studies have been proved [42].

Onion (Allium cepa Linn) is an important allium species commonly used as ingredients in many dishes for flavour and taste enhancement [32]. Onion is rich in two significant bioactive compounds; organosulfur compounds and flavonoids $[36,40]$. It possesses antioxidant, anti-inflammatory, and antimicrobial properties [15]. Its role in lowering the lipid peroxide levels had been reported by some researchers [33] but denied by others [25].

Although many researchers studied the protective effect of NS oil on lung damage-induced by many harmful agents [43], up to our knowledge, no researchers studied the protective potential of NS oil on lung damage induced by nicotine. Moreover, few researchers were concerned by the protective effect of onion oil on lung damage induced by nicotine $[19,20]$. So, the present work aimed to compare the protective effect of NS and onion extract on nicotine-induced lung damage in rats. Furthermore, the antioxidant and anti-lipid peroxidation potentials of both agents on nicotine-induced lung damage were studied.

\section{MATERIALS AND METHODS}

\section{Chemical}

Nicotine hydrogen bitartrate (Sigma Aldrich Co., St. Louis, Mo) was dissolved in $0.9 \%$ saline and neutralised to $\mathrm{PH} 7.2$ with $\mathrm{NaOH}$. The treatment was given at a dose of $2.5 \mathrm{mg} / \mathrm{kg}$ subcutaneously once daily for 5 days per week/18 weeks [11]. Selection of dose of nicotine was constructed on the previous studies and doses commonly used by other laboratories [11] and the selection of time schedule was based on that more than 20 days of nicotine injection leads to oxidative stress and tissue injury [38, 39].

Red onion was used because of its reported high antioxidant potentials [32]. It was dressed and frozen $\left(4^{\circ} \mathrm{C}\right) .100 \mathrm{~mL}$ of distilled water per $100 \mathrm{~g}$ of onion were added and crushed in a mixing machine. The resultant mixture was squeezed and filtered [32]. The onion extract was given orally once/day in a dose of $50 \mathrm{mg} / \mathrm{kg}$ [44]. This dose is nontoxic and has few ill effects [44].

The NS oil was given at a daily dose of $0.1 \mathrm{~g} / \mathrm{kg}$ orally. The dosage was chosen based on human NS oil consumption which is equal to $2 \mathrm{~g} /$ day; $0.1 \mathrm{~g} / \mathrm{kg} /$ day was accepted as the corresponding dose in the rat [12].

All chemicals were purchased from Cap Pharma Company, Egypt.

\section{Animals}

Thirty-six Sprague-Dawley adult male albino rats were acclimatised in the laboratory for a period of 2 weeks before carrying out the experiment. The sample size was based according to the resource equation method $[8,14]$. The rats were kept in cages with a temperature around $\left(26 \pm 2^{\circ}\right)$ with $12 \mathrm{~h}$ light and $12 \mathrm{~h}$ dark cycle.

The rats were observed daily for signs of morbidity and mortality. The general toxicological data were recorded including the motility, food and water consumption, health status and body weight gain. The study was approved by the Ethics Committee, Faculty of Medicine, Cairo University (321/2018). The study 
was performed according to the ethical standards of the National Institutes of Health guide for the care and use of Laboratory Animals ( $8^{\text {th }}$ edition, revised in 2011).

\section{Experimental design}

At the beginning of the study, the rats' age was approximately 7 months. They were divided into six groups; each one consisted of 6 rats: one negative control group, two positive control groups (oral onion and oral NS), nicotine-treated group, onion extract-treated group (concomitant nicotine and onion extract) and NS-treated group (concomitant nicotine and NS oil). The antioxidants of the positive control groups were given orally via a syringe with a gavage needle according to the drug administration protocol. In the last two groups, the antioxidants were administered orally following the nicotine injection. All rats were sacrificed after 18 weeks.

\section{Tissue sampling}

The lung was dissected and fixed immediately in $10 \%$ formalin saline. To randomise selection, the entire lung was cut starting at the superior border; every $10^{\text {th }}$ section ( $5 \mu \mathrm{m}$ thick) was put aside for staining.

\section{Preparation of tissue extracts}

A portion of lungs was homogenised in 10 volumes (1:10; w/v) of ice-cold $10 \mathrm{mM}$ phosphate buffered saline (PBS, pH 7.4) in an Ultra Turrax tissue homogenizer for $30 \mathrm{~s}$. Homogenates were centrifuged at $10,000 \mathrm{rpm}$ for $10 \mathrm{~min}$ at $4^{\circ} \mathrm{C}$. The supernatant was pipetted into clean centrifuge tubes and stored in aliquots $\left(-80^{\circ} \mathrm{C}\right)$ until analysis [11].

\section{Light microscopic study}

The lung sections were stained with haematoxylin and eosin (H\&E) stain [5]. The stained sections were examined and photographed using a Canon digital camera (Canon, Tokyo, Japan) connected to an IBM computer system.

\section{Transmission electron microscopic study}

Small pieces of the lung from each rat were fixed in 2\% paraformaldehyde and $2 \%$ glutaraldehyde solution in $0.1 \mathrm{~mol} / \mathrm{L} \mathrm{PBS} \mathrm{pH} 7.2$ and kept in the refrigerator overnight, rinsed in $0.1 \mathrm{~mol} / \mathrm{LPS}$ and post fixed in phosphate-buffered $1 \%$ osmium tetroxide. Fixation was followed by dehydration and embedding in epoxy resins. Semithin cross-sections were stained with $1 \%$ toluidine blue. Ultrathin sections $(50-60 \mathrm{~nm})$ were stained with uranyl acetate and lead citrate. These sections were examined and photographed using a Jeom-1400 transmission electron microscope (JEOL Ltd./Japan a Joel).

\section{Oxidative/antioxidative markers}

Malondialdehyde (MDA), a marker for lipid peroxidation, was measured by monitoring thiobarbituric reactive substances (TBARS) formation. The concentration of MDA was calculated and expressed in nanomoles per milligram of protein.

Superoxide dismutase (SOD) activity was determined at room temperature according to the modified Misra and Fridovich's method [31].

The lung catalase (CAT) activity was evaluated at room temperature according to the Aebi's method [1]. One unit of CAT activity is equal to the $\mu$ moles of $\mathrm{H}_{2} \mathrm{O}_{2}$ degraded per minute per milligram of protein ( $\mu \mathrm{mol} / \mathrm{H}_{2} \mathrm{O}_{2}$ utilised $/ \mathrm{min} / \mathrm{mg}$ protein).

Glutathione (GSH) was measured spectrophotometrically in the bronchoalveolar lavage fluid (BALF) and lung tissue [34]. The concentration of the lung GSH was calculated using the standard curve and expressed per milligram of protein. The level of epithelial lining fluid GSH (ELF GSH) was expressed $\mu \mathrm{M}$.

The protein concentration in the lung homogenate was measured by Bradford's method using bovine serum albumin as standard [7].

\section{Statistical analysis}

Statistical analysis was performed using statistical package for the social sciences (SPSS) version 21.0 (IBM Corporation, Somers, NY, USA) statistical software. Data were expressed as means \pm standard deviation (SD). Statistical evaluation was done using one-way analysis of variance (ANOVA) followed by Bonferroni pairwise comparisons. Significance was considered when the $p$-value was less than 0.05 .

The percentage of increase or decrease (difference) of all study parameters were calculated with the following formula:

Percentage of difference $=$ (mean difference value between two groups) / (value of the compared group) $\times 100$.

\section{RESULTS}

General characteristic data of the rats (Table 1)

No mortalities were detected in the different studied groups. The motility, food and water consumption and health status were good. 
Table 1. The body weight and protein of lung in the studied groups at the end of the study

\begin{tabular}{lcc}
\hline Group & Body weight [g] & Protein [g/100 g of lung] \\
\hline Control & $280.0 \pm 7.9$ & $6.8 \pm 0.2$ \\
Nicotine & $237.0 \pm 5.7^{* \# \$}$ & $6.0 \pm 0.2^{* \$}$ \\
Nicotine-onion & $257.2 \pm 7.0^{* \%}$ & $6.2 \pm 0.2^{* \$}$ \\
Nicotine-NS & $270.0 \pm 7.9^{\%}$ & $6.6 \pm 0.1^{\% \#}$ \\
\hline
\end{tabular}

Data are shown as mean \pm standard deviation; ${ }^{*}$ significant in relation to the control group; " significant in relation to the nicotine-onion group; 'significant in relation to the nicotine-NS group; "significant in relation to the nicotine group; NS — Nigella sativa

The body weight at the beginning of the study was $220.0 \pm 5.4 \mathrm{~g}$. At the end of the study, the animals of the different studied groups gained weight. The body weight in the nicotine-treated group was $15 \%$ less than the weight of the control group. More weight gain was observed in the onion extract and NS-treated groups.

The lung protein decreased in the nicotine group by $11.7 \%$ in relation to that of the control group. Higher elevation was observed with the use of onion extract and NS $(3.3 \%$ and $10 \%$ higher than that of the nicotine-treated group).

\section{Levels of lipid peroxidation (Table 2)}

Administration of nicotine in the study promoted lipid peroxidation. The level of MDA in lung homogenates was significantly increased in the nicotine group by $86.66 \%$ compared with the rate of the control groups. With the use of onion extract and NS, the level of MDA decreased by $17.85 \%$ and $35.71 \%$ compared with the nicotine group.

\section{Levels of antioxidant markers (Table 2)}

Administration of nicotine decreased the level of the antioxidant markers. Compared to the control rats, nicotine-treated animals showed a significant decrease in the levels of SOD and CAT by $58.33 \%$ and $26.41 \%$, respectively. With the use of onion extract and NS, the level of SOD increased by $57.14 \%$ and $122 \%$, and the level of CAT increased by $16 \%$ and $29.48 \%$, respectively compared with the level of the nicotine group. The increase was more prominent in the NS-treated group. The level of both markers in the NS-treated group reached nearly the level markers of the control group.

Furthermore, compared to the control rats, the nicotine-treated animals showed a significantly lower level (53.15\% and $44.44 \%$ ) of the lung GSH and ELF GSH. With the use of onion extract and NS, the level of the lung GSH increased by $49.33 \%$ and $91.11 \%$, and the level of the ELF GSH increased by $37.84 \%$ and $69.23 \%$, respectively compared with their levels of the nicotine group. The level of both markers in the NS-treated group reached nearly the level markers of the control groups.

\section{The general structure of the lung as shown by H\&E staining}

The lung of the control groups exhibited the normal histological architecture (Fig. 1A, B).

The lung in the nicotine-treated group exhibited emphysematous air spaces, collapsed corrugated alveoli with ruptured interalveolar septa in some specimen (4/6) and thickened septa in the others (2/6) (Fig. 1C, D). In addition, massive congestion, ex-

Table 2. Lipid peroxidation, oxidative/antioxidative markers in the studied groups

\begin{tabular}{|c|c|c|c|c|c|}
\hline Group & $\begin{array}{c}\text { MDA } \\
\text { [nmol/mg protein] }\end{array}$ & $\begin{array}{c}\text { SOD } \\
{[\mathrm{U} / \mathrm{mg} \text { protein }]}\end{array}$ & $\begin{array}{c}\mathrm{CAT}\left[\mu \mathrm{mo} / \mathrm{H}_{2} \mathrm{O}_{2}\right. \\
\text { utilised } / \mathrm{min} / \mathrm{mg} \text { protein] }\end{array}$ & $\begin{array}{c}\text { ELF GSH } \\
{[\mu \mathrm{M}]}\end{array}$ & $\begin{array}{c}\text { Lung GSH } \\
\text { [nmol/mg protein] }\end{array}$ \\
\hline Control & $0.15 \pm 0.01$ & $16.8 \pm 1.5$ & $42.4 \pm 2.1$ & $96.0 \pm 9.6$ & $117.0 \pm 12.0$ \\
\hline Nicotine & $0.28 \pm 0.02^{* \# \$}$ & $7.0 \pm 1.6^{*} \# \$$ & $31.2 \pm 1.9^{* \# \$}$ & $45.0 \pm 7.9^{* \# \$}$ & $65.0 \pm 7.9^{* \# \$}$ \\
\hline Nicotine-onion & $0.23 \pm 0.02^{* \% s}$ & $11.0 \pm 1.61^{* 0 / s}$ & $36.2 \pm 1.3^{* 0 / 8 s}$ & $67.2 \pm 5.6^{* \% s}$ & $89.6 \pm 7.3^{* \% \%}$ \\
\hline Nicotine-NS & $0.18 \pm 0.02 \%$ & $15.6 \pm 1.5^{\% \#}$ & $40.4 \pm 1.1^{\% \#}$ & $86.0 \pm 4.2^{\% \#}$ & $110.0 \pm 7.9^{* \% \#}$ \\
\hline Versus control & 0.060 & 0.001 & 0.446 & 0.225 & $1.00^{*}$ \\
\hline Versus nicotine & $0.000^{*}$ & $0.000^{*}$ & $0.000^{*}$ & $0.000^{*}$ & $0.000^{*}$ \\
\hline Versus nicotine-onion & $0.000^{*}$ & $0.001 *$ & $0.006^{*}$ & $0.004^{*}$ & $0.015^{*}$ \\
\hline
\end{tabular}

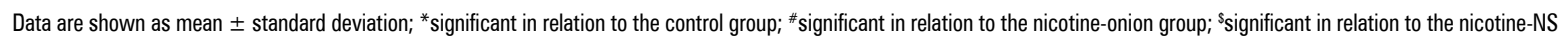
group; "significant in relation to the nicotine group; NS — Nigella sativa; MDA — lung malondialdehyde; SOD — superoxide dismutase activity; CAT — catalase; GSH — glutathione; ELF GSH — epithelial lining fluid GSH 


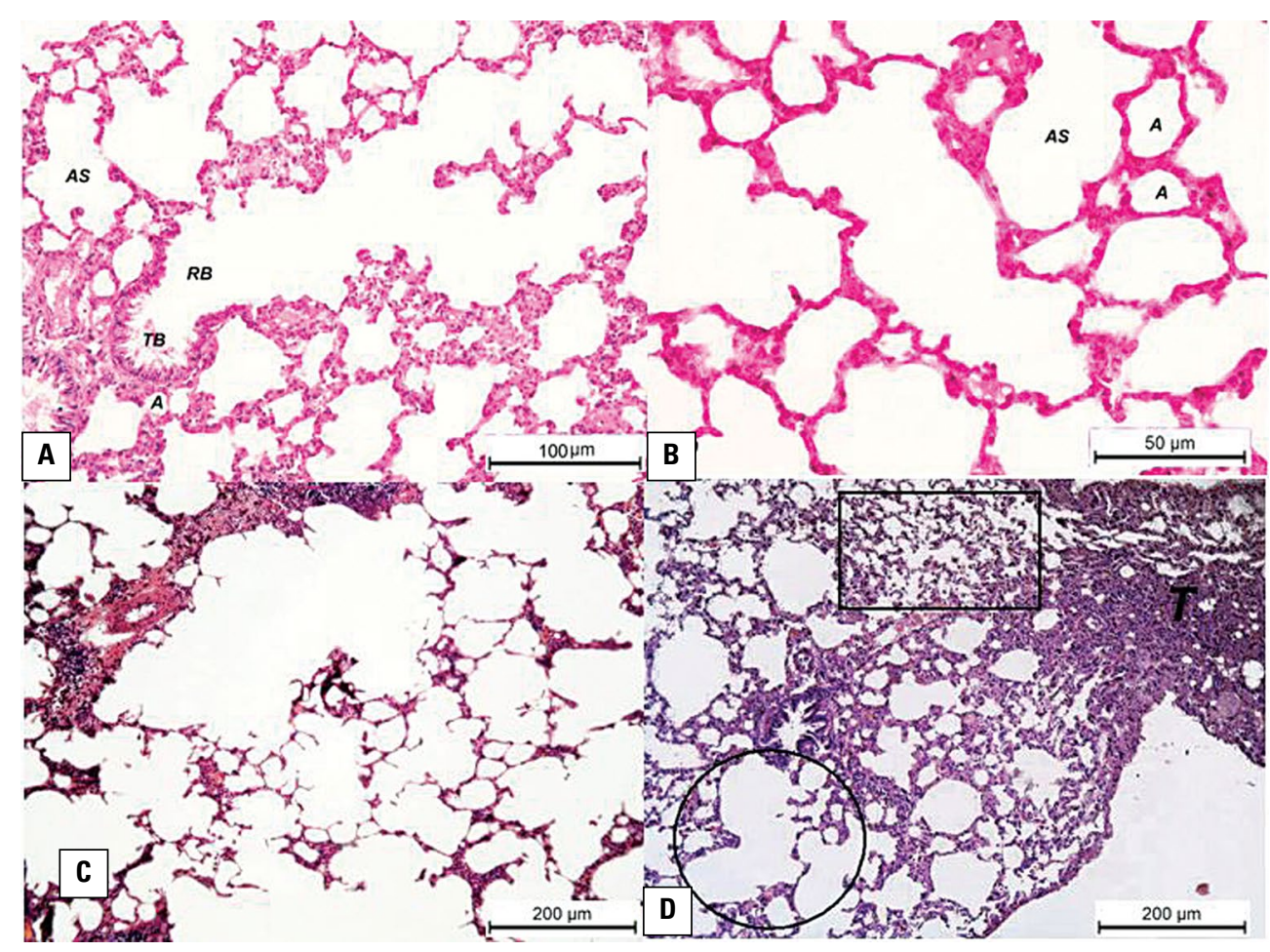

Figure 1. Lung morphology in the control and nicotine-treated groups (haematoxylin-eosin); A, B. Control rats: alveolar sac (AS), respiratory bronchiole (RB), terminal bronchiole (TB) and alveoli $(A)(\times 200$ and $\times 400$, respectively); C, D. Nicotine-treated group $(\times 100)$; C. Emphysematous air spaces with ruptured interalveolar septa; D. Emphysematous air spaces (encircled area), collapsed corrugated alveoli (boxed area) and thickened septa (T).

travasation of red blood cells, inflammatory cellular infiltration and fluid exudate (3/6) were seen in this group (Fig. 2A-D).

Much improvement was observed in the onion extract-treated group despite the presence of residual pathological nicotine affection of the lung such as thickened interalveolar septa, congested capillaries and massive foamy macrophages (2/6) (Fig. 3A, B). The lung in the NS-treated group showed the nearly normal architecture with slight congestion (5/6) (Fig. 3C, D).

\section{Semithin and ultrathin structure of the lung}

The semithin sections in the lung of the control group displayed the normal architecture (Fig. 4A). Mast cells, plasma cells, and fluid exudate were detected in the nicotine-treated group (2/6) (Fig. 4B). Foamy macrophages $(1 / 6)$ were seen in the onion extract-treated group. Nearly normal lung architecture $(5 / 6)$ was observed in the NT-treated group.

The ultrathin sections of the lung of the nicotine-treated group exhibited the presence of interalveolar exudate, multiple pneumocytes II, fibroblasts, macrophages and collagen (5/6) (Fig. 5A, B). Many affections of the lung of the onion extract-treated group such as the presence of collagen and mast cells were detected (3/6) (Fig. 5C). The NS-treated group showed collagen with few lysosomes and macrophages (1/6) (Fig. 5D).

\section{DISCUSSION}

The body weight in the nicotine-treated group was significantly lower than the weight of the control group. More weight gain was observed in the onion extract and NS-treated groups; however, both groups did not reach the weight of the control group. The weight-reducing effect of smoking is well documented which may be attributed to nicotine's appetite suppression property and/or its metabolism stimulation property with more caloric burn [9].

Chronic nicotine administration has a deleterious effect on the lung. The emphysema with collapsed corrugated accompanied the nicotine administration were the consequences of the observed alveolar damage [11]. The congestion, haemorrhage, and oedema were the consequences of the increased vascular permeability, pulmonary microvascular leakage and the loss of the pulmonary capillary endothelium [11]. Such findings could indicate damage to the cellular clearance systems and pulmonary lymphatic system [11]. 


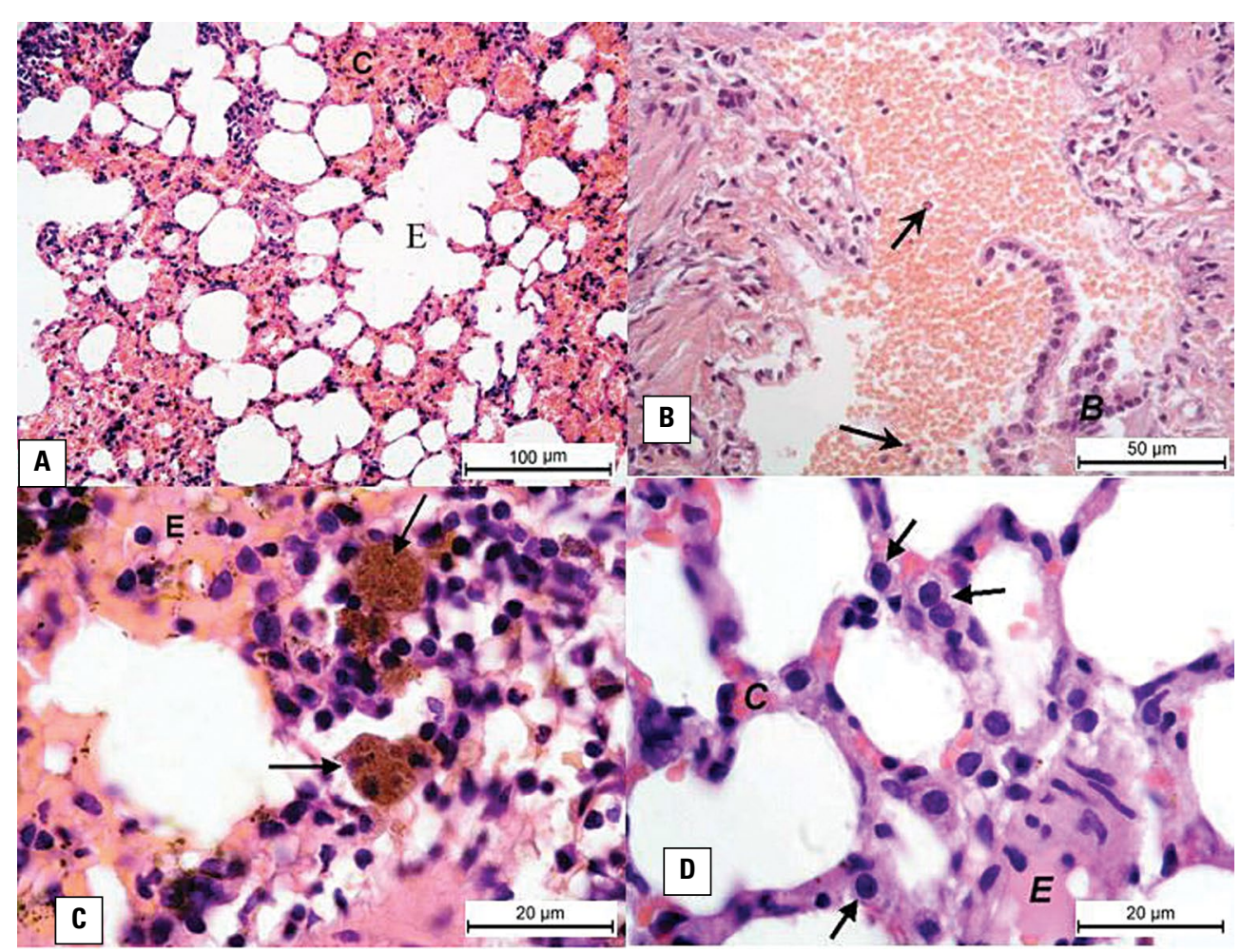

Figure 2. Lung morphology in the nicotine-treated group (haematoxylin-eosin); A. Massive congestion (C) with emphysematous air spaces (E) (haematoxylin-eosin; $\times 200$ ); B. Disrupted bronchiole (B), extravasated red blood cells (arrowheads) and inflammatory cells (arrows) $(\times 400)$; C. Fluid exudate $(E)$ and macrophages (arrows) $(\times 1000)$; D. Increased number of pneumocyte II (arrows), congested capillaries $(C)$ and fluid exudate $(E)(\times 1000)$.

In addition, interstitial inflammatory cellular infiltration in the nicotine-treated group was attributed to nicotine triggering inflammatory cascade in the airway epithelium ensuing the production of numerous strong cytokines and chemokines, with the recruitment of neutrophils and macrophages to the airways [24].

The previous histopathological changes with the increased level of the lipid peroxidation (oxidant; MDA) and the decreased levels of the pulmonary antioxidants reflect an oxidative stress. Oxidative stress is defined as the shift in the balance between oxidants and antioxidants in the favour of oxidants [6]. The chronic exposure to nicotine (18 weeks) besides its high concentrations $(2.5 \mathrm{mg} / \mathrm{kg})$ were the two inductive factors causing such oxidative stress $[16,38$, 39]. Low concentration of nicotine $(10 \mu \mathrm{M})$ is actually preventive of oxidative stress as the nicotine at such dose inhibits the $\mathrm{H}_{2} \mathrm{O}_{2}$ induced lipid peroxidation [16].

The resultant oxidative stress in the lung generates oxygen free radical (reactive oxygen species [ROS]) which has the ability to react with numerous biomolecules in the cell, including proteins, lipids, and nucleic acids, which caused oxidative damage and eventually lead to cell death [29]. Such destructive species can be scavenged by the host antioxidant defence system. The first antioxidant defence is SOD which catalyses the dismutation of $\mathrm{O}_{2}^{-}$to $\mathrm{H}_{2} \mathrm{O}_{2}$ and $\mathrm{O}_{2}$ [23]. The second antioxidant defence is CAT which scavenges $\mathrm{H}_{2} \mathrm{O}_{2}$ generated by SOD and converts it to $\mathrm{H}_{2} \mathrm{O}$ and $\mathrm{O}_{2}$ [35]. GSH is an important antioxidant defence that offers protection against free radicals, peroxides and toxic compounds [30]. Chronic nicotine administration decreased the activities of the free radical scavenging enzymes SOD, CAT, and GSH which may be due to the enhanced utilisation of these antioxidants during detoxification of nicotine. This results in increased generation of $\mathrm{O}_{2}{ }^{-}$and $\mathrm{H}_{2} \mathrm{O}_{2}$ which in turn results in generation of $\mathrm{OH}^{-}[17]$. The generation of $\mathrm{OH}^{-}$participates in many toxic reactions [17].

Enhanced lipid peroxidation (increased MDA level) was observed in the nicotine-treated group. The higher level of lipid peroxidation products in the lung of the nicotine-treated rats may be due to nicotine ROS [11]. The increased lipid peroxidation led to a disruption of the pulmonary cell membranes' 


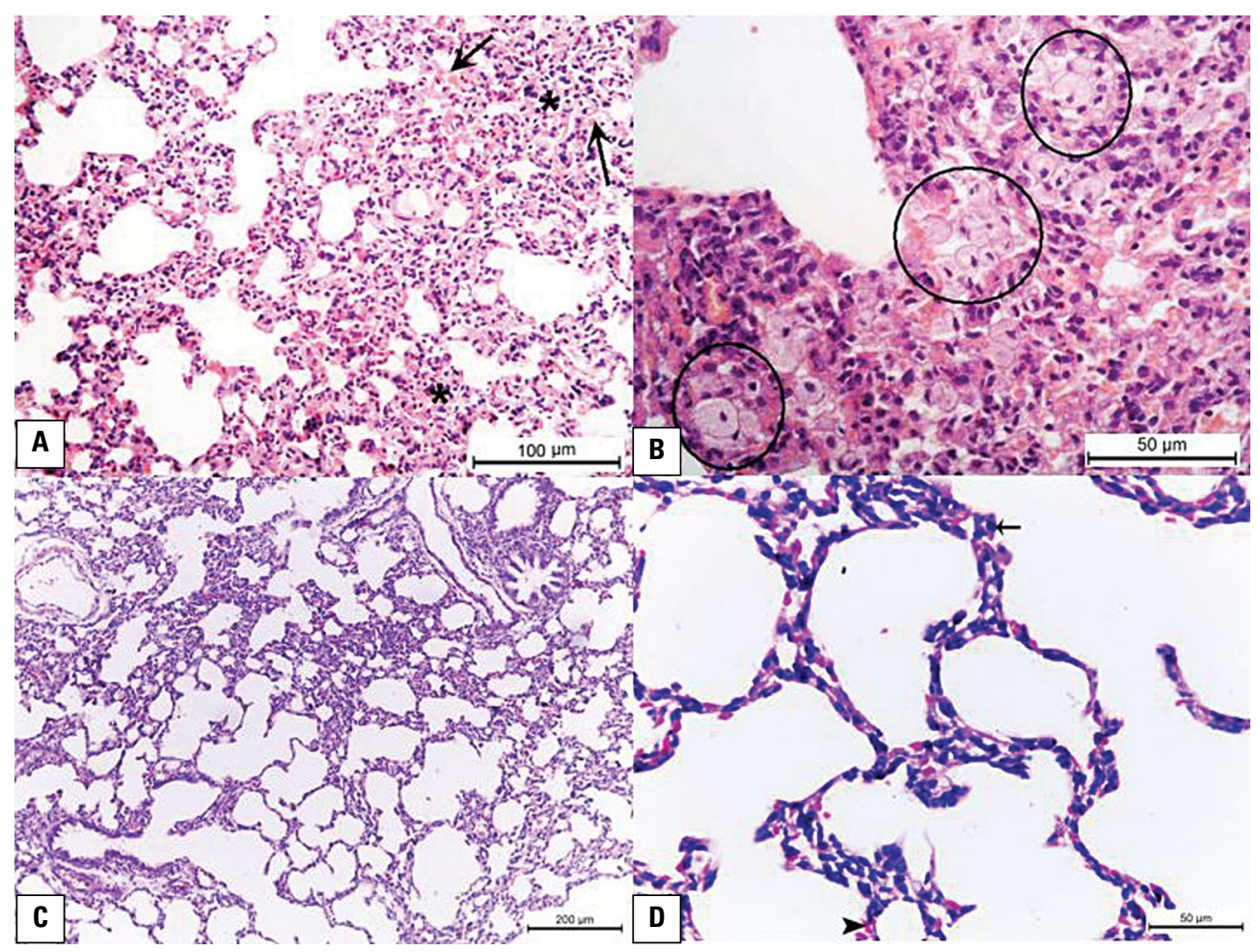

Figure 3. Lung morphology in the onion extract and nigella-treated groups (haematoxylin-eosin); A, B. Onion extract-treated group; A. Thickened interalveolar septa $\left(^{*}\right)$ with congested capillaries (arrows) $(\times 200)$; B. Massive foamy macrophages (encircle) within thickened septa $(\times 400)$; C, D. Nigella-treated group; C. Normal lung architecture with slight congestion ( $\times 100)$; D. Normal lung architecture with slight congestion (arrowhead) and pneumocyte II (arrow) $(\times 400)$.

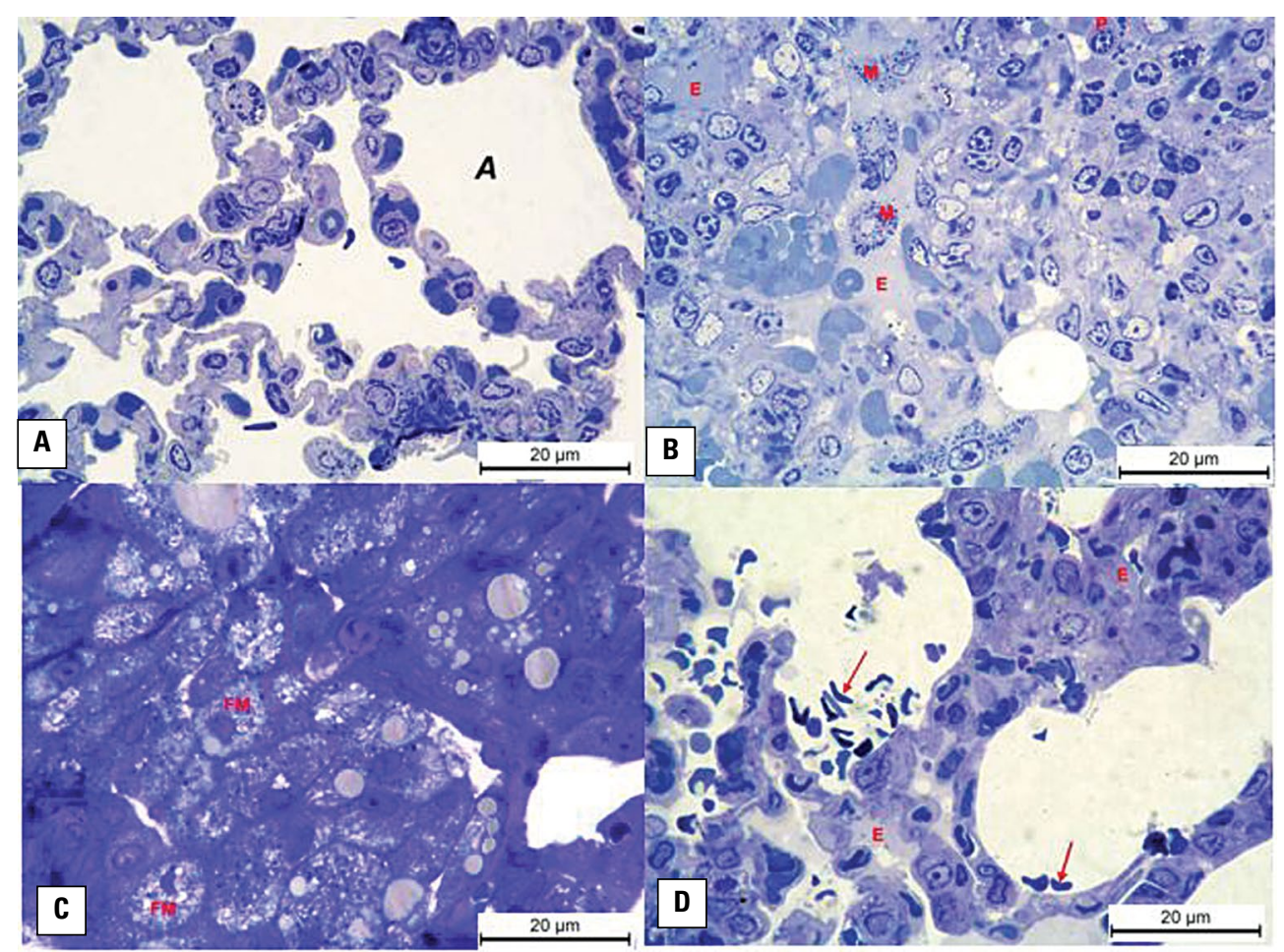

Figure 4. Semithin sections in the lung of the different treated groups (toluidine blue; $\times 1000$ ); A. Control group: normal lung architecture. Note the alveoli (A); B. Nicotine-treated group: mast cells $(M)$, plasma cells $(P)$ and fluid exudate (E); C. Onion-treated group: foamy macrophages (FM); D. Nigella-treated group: normal lung architecture with extravasated red blood cells (arrows) and exudate (E). 


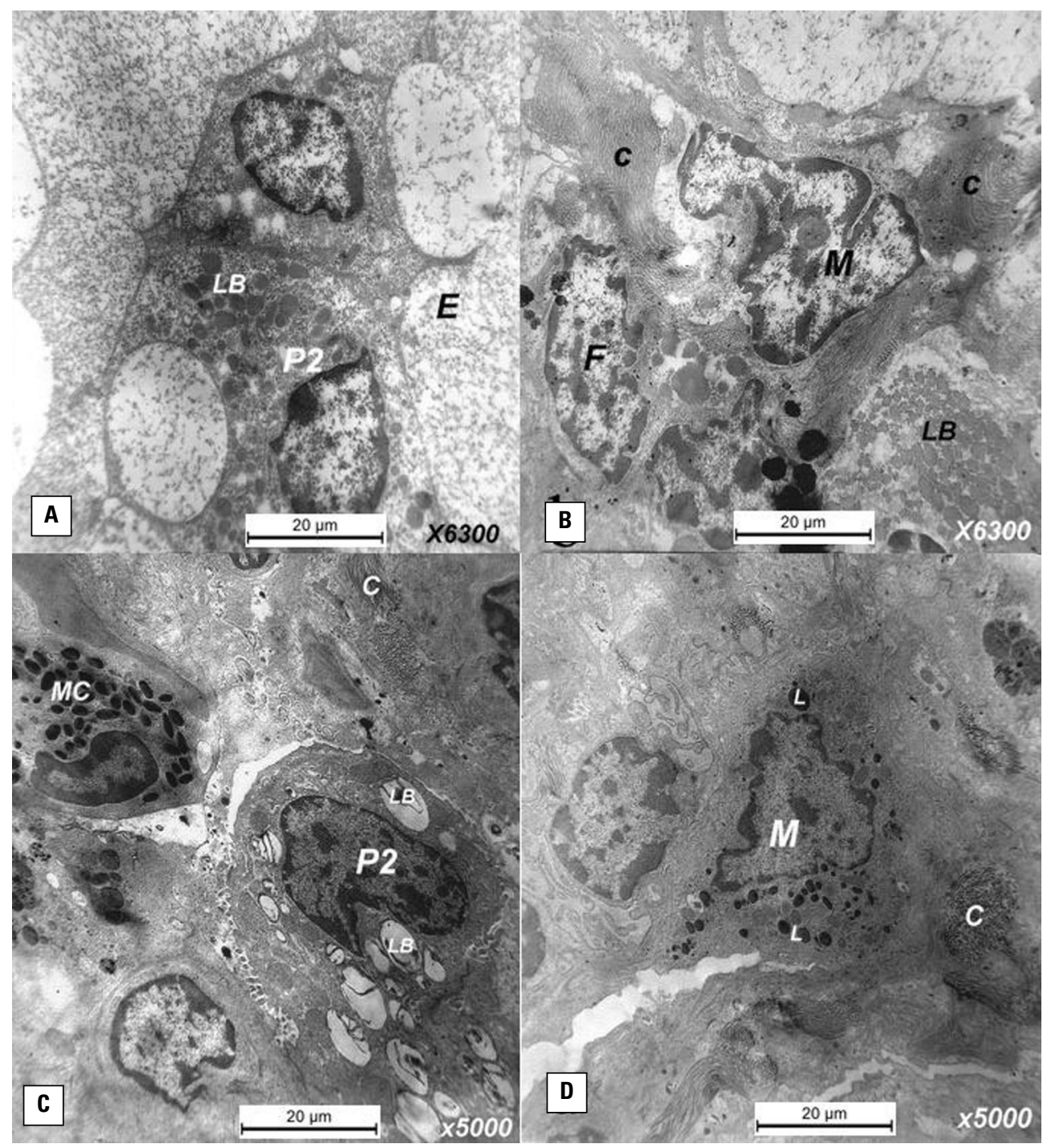

Figure 5. Ultrathin in the lung of the different treated groups; A, B. Nicotine-treated group: pneumocyte II (P2) with lamellar bodies (LB), exudate $(E)$ in interalveolar spaces, collagen $(C)$, a fibroblast $(F)$ and a macrophage $(M)(\times 6300)$; C. Onion-treated group: collagen (C), lamellar bodies (LB) in a pneumocyte II (P2) and a mast cell $(\mathrm{MC})(\times 5000)$; D. Nigella-treated group: collagen (C), lysosomes (L) in a macrophage (M) $(\times 5000)$.

integrity and to the cytoplasmic enzyme's leakage [11]. Lipid peroxidation can be used as an index for measuring the cell membrane damage that occurs in because of ROS [22].

Much improvement of the structure and ultrastructure of the lung was observed in the onion extract-treated group despite the presence of residual pathological affection. In contrary, the lung of the NS-treated group showed nearly normal architecture.

The first mechanism through which the onion extract and NS protected the lung against the destructive effect of nicotine was through suppression of the lipid peroxidation. Compared to the nicotine group, the level of lung MDA significantly decreased in the onion extract group with double the descent in the NS-treated group. The anti-lipid peroxidation role of NS is attributed to thymoquinone (TQ) which is the main component of the NS oil [27]. Many researchers proved the anti-lipid peroxidation role of TQ. TQ has a protective effect on the membrane lipid peroxidation in hepatocytes [27] and on the lipid peroxidation during ischaemia-reperfusion injury in the rat hippocampus [21]. In addition, TQ decreased the hepatic and the erythrocyte lipid peroxidation [18]. On the other hand, concerning the lipid peroxidation role of onion extract, controversy in the literature was 
observed. Lipid peroxidation-reducing effect of the onion extract against $\mathrm{Cd}$-induced testicular oxidative damage in rats was proved by some researchers [10]. Park et al. [33] reported that onion supplementation is valuable for lowering lipid peroxide levels to the aged rats. However, in this study, the onion peel extract supplementation had no effect on lipid peroxidation parameters or inhibitory capacity against leukocyte DNA damage [33].

The second mechanism through which the onion extract and NS protected the lung against the destructive effect of nicotine was through the antioxidant mechanism. All the antioxidant enzymes used in our study (SOD, CAT, lung GSH and ELF GSH) increased in the onion extract and NS-treated groups with the highest level was in the NS-treated group. The antioxidant role of NS is also attributed to TQ [4]. The anti-oxidant TQ might be attributed to its scavenging activity against several ROS including $\mathrm{O}_{2}^{-} \mathrm{OH}^{-}$and 01 [28]. In addition, many researchers proved the protective effect of TQ on numerous organs against oxidative damage induced by a diversity of free radical-generating agents [10]. Similarly, the antioxidative effect of the onion extract was proved in many works of literature [26] and against Cd-induced testicular oxidative damage in rats [32].

\section{CONCLUSIONS}

In conclusion, NS and onion extract attenuate the pathological effect of nicotine in the lung rats through antioxidative and anti-lipid peroxidative mechanisms with higher protection to Nigella sativa.

\section{REFERENCES}

1. Aebi H. Catalase in vitro. Methods Enzymol. 1984; 105: 121-126.

2. Al-Ghamdi MS. The anti-inflammatory, analgesic and antipyretic activity of Nigella sativa. J Ethnopharmacol. 2001; 76(1): 45-48, doi: 10.1016/s0378-8741(01)00216-1.

3. Ali BH, Blunden G. Pharmacological and toxicological properties of Nigella sativa. Phytother Res. 2003; 17(4): 299-305.

4. Badary OA, Gamal El-Din AM. Inhibitory effects of thymoquinone against 20-methylcholanthrene-induced fibrosarcoma tumorigenesis. Cancer Detect Prev. 2001; 25(4): 362-368.

5. Bancroft JD, Gamble M. Theory and practice of histological techniques. 6th edn. Churchill Livingstone/Elsevier, Philadelphia 2008.

6. Birben E, Sahiner U, Sackesen C, et al. Oxidative stress and antioxidant defense. World Allergy Org J. 2012; 5(1): 9-19, doi: 10.1097/wox.0b013e3182439613.

7. Bradford M. A rapid and sensitive method for the quantitation of microgram quantities of protein utilizing the principle of protein-dye binding. Analytical Bioch. 1976; 72(1-2): 248-254, doi: 10.1016/0003-2697(76)90527-3.

8. Charan J, Biswas T. How to calculate sample size for different study designs in medical research? Indian J Psychol Med. 2013; 35(2): 121-126, doi: 10.4103/02537176.116232, indexed in Pubmed: 24049221.

9. Courtemanche C, Tchernis R, Ukert B. The effect of smoking on obesity: Evidence from a randomized trial. J Health Econ. 2018; 57: 31-44.

10. Darakhshan S, Bidmeshki Pour A, Hosseinzadeh Colagar A, et al. Thymoquinone and its therapeutic potentials. Pharmacol Res. 2015; 95-96: 138-158, doi: 10.1016/j. phrs.2015.03.011, indexed in Pubmed: 25829334.

11. Dhouib $H$, Jallouli $M$, Draief $M$, et al. Oxidative damage and histopathological changes in lung of rat chronically exposed to nicotine alone or associated to ethano. Pathol Biol (Paris). 2015; 63(6): 258-67.

12. Dollah MA, Parhizkar S, Izwan M. Effect of Nigella sativa on the kidney function in rats. Avicenna J Phytomed. 2013; 3(2): 152-158.

13. El-Sokkary GH, Cuzzocrea S, Reiter RJ. Effect of chronic nicotine administration on the rat lung and liver: beneficial role of melatonin. Toxicology. 2007; 239(1-2): 60-67.

14. Festing MF, Altman DG. Guidelines for the design and statistical analysis of experiments using laboratory animals. ILAR J. 2002; 43(4): 244-58.

15. Gorinstein S, Leontowicz $\mathrm{H}$, Leontowicz $\mathrm{M}$, et al. Comparison of the main bioactive compounds and antioxidant activities in garlic and white and red onions after treatment protocols. J Agric Food Chem. 2008; 56(12): 4418-4426.

16. Guan ZZ, Yu WF, Nordberg A. Dual effects of nicotine on oxidative stress and neuroprotection in PC12 cells. Neurochem Int. 2003; 43(3): 243-249.

17. Halliwell B, Gutteridge J. Free radicals and antioxidant protection: mechanisms and significance in toxicology and disease. Hum Toxicol. 1988; 7(1): 7-13, doi: 10.1177 /096032718800700102.

18. Harzallah HJ, Grayaa R, Kharoubi W, et al. Thymoquinone, the Nigella sativa bioactive compound, prevents circulatory oxidative stress caused by 1,2-dimethylhydrazine in erythrocyte during colon postinitiation carcinogenesis. Oxid Med Cell Longev. 2012; 854065.

19. Helen A, Krishnakumar K, Vijayammal PL, et al. Antioxidant effect of onion oil (Allium cepa. Linn) on the damages induced by nicotine in rats as compared to alpha-tocopherol. Toxicol Lett. 2000; 116(1-2): 61-68, indexed in Pubmed: 10906423.

20. Helen A, Rajasree CR, Krishnakumar K, et al. Antioxidant role of oils isolated from garlic (Allium sativum Linn) and onion (Allium cepa Linn) on nicotine-induced lipid peroxidation. Vet Hum Toxicol. 1999; 41(5): 316-319.

21. Hosseinzadeh $H$, Parvardeh $S$, Asl MN, et al. Effect of thymoquinone and Nigella sativa seeds oil on lipid peroxidation level during global cerebral ischemia-reperfusion injury in rat hippocampus. Phytomedicine. 2007; 14(9): 621-627.

22. Husain K, Scott BR, Reddy SK, et al. Chronic ethanol and nicotine interaction on rat tissue antioxidant defense system. Alcohol. 2001; 25(2): 89-97.

23. Husain K, Somani SM. Interaction of exercise training and chronic ethanol ingestion on testicular antioxidant system in rat. J Appl Toxicol. 1998; 18(6): 421-429. 
24. Kalpana C, Menon VP. Modulatory effects of curcumin on lipid peroxidation and antioxidant status during nicotine-induced toxicity. Pol J Pharmacol. 2004; 56(5): 581-586.

25. Kim J, Cha YJ, Lee KH, et al. Effect of onion peel extract supplementation on the lipid profile and antioxidative status of healthy young women: a randomized, placebo-controlled, double-blind, crossover trial. Nutr Res Pract. 2013; 7(5): 373-379.

26. Lisanti A, Formica V, lanni F, et al. Antioxidant activity of phenolic extracts from different cultivars of Italian onion (Allium cepa) and relative human immune cell proliferative induction. Pharm Biol. 2016; 54(5): 799-806.

27. Mansour MA, Ginawi O, El-Hadiyah T. Effects of volatile oil constituents of Nigella sativa on carbon tetrachloride-induced hepatotoxicity in mice: evidence for antioxidant effects of thymoquinone. Res Commun Mol Pathol Pharmacol. 2001; 110(3-4): 239-251.

28. Mansour MA, Nagi MN, El-Khatib AS. Effects of thymoquinone on antioxidant enzyme activities, lipid peroxidation and DT-diaphorase in different tissues of mice: a possible mechanism of action. Cell Biochem Funct. 2002; 20(2): 143-151.

29. McCord JM. Human disease, free radicals, and the oxidant/ antioxidant balance. Clin Biochem. 1993; 26(5): 351-357, indexed in Pubmed: 8299205.

30. Meister A. Glutathione, ascorbate, and cellular protection. Cancer Res. 1994; 54(7 Suppl): 1969s-1975s.

31. Misra HP, Fridovich I. The role of superoxide anion in the autoxidation of epinephrine and a simple assay for superoxide dismutase. J Biol Chem. 1972; 247(10): 3170-3175.

32. Ola-Mudathir KF, Suru SM, Fafunso MA, et al. Protective roles of onion and garlic extracts on cadmium-induced changes in sperm characteristics and testicular oxidative damage in rats. Food Chem Toxicol. 2008; 46(12): 3604-3611.

33. Park J, Kim J, Kim MK. 'Onion flesh and onion peel enhance antioxidant status in aged rats. J Nutr Sci Vitaminol (Tokyo). 2007; 53(1): 21-29.

34. Rahman I, Kode A, Biswas SK. Assay for quantitative determination of glutathione and glutathione disulfide levels using enzymatic recycling method. Nat Protoc. 2006; 1(6): 3159-3165.

35. Ribière $\mathrm{C}$, Hininger $\mathrm{I}$, Rouach $\mathrm{H}$, et al. Effects of chronic ethanol administration on free radical defence in rat myocardium. Biochem Pharmacol. 1992; 44(8): 1495-1500.

36. Rohn S, Buchner N, Driemel G, et al. Thermal degradation of onion quercetin glucosides under roasting conditions. J Agric Food Chem. 2007; 55(4): 1568-1573.

37. Schweitzer KS, Chen SX, Law S. Endothelial disruptive proinflammatory effects of nicotine and e-cigarette vapor exposures. Am J Physiol Lung Cell Mol Physiol. 2015; 309(2): L175-L187.

38. Sener G, Kapucu C, Paskaloglu K, et al. Melatonin reverses urinary system and aorta damage in the rat due to chronic nicotine administration. J Pharm Pharmacol. 2004; 56(3): 359-266.

39. Sener G, Ozer Sehirli A, Cetinel S, et al. Taurine treatment protects against chronic nicotine-induced oxidative changes. Fundam Clin Pharmacol. 2005; 19(2): 155-164.

40. Slimestad R, Fossen T, Vågen IM. Onions: a source of unique dietary flavonoids. J Agric Food Chem. 2007; 55(25): 10067-10080, doi: 10.1021/jf0712503, indexed in Pubmed: 17997520.

41. Soto-Otero R, Méndez-Alvarez E, Hermida-Ameijeiras $A$, et al. Effects of (-)-nicotine and (-)-cotinine on 6-hydroxydopamine-induced oxidative stress and neurotoxicity: relevance for Parkinson's disease. Biochem Pharmacol. 2002; 64(1): 125-135.

42. Tavakkoli A, Ahmadi A, Razavi BM, et al. Black Seed. Iran J Pharm Res. 2017; 16(Suppl): 2-23.

43. Tayman C, Cekmez F, Kafa IM, et al. Protective Effects of Nigella sativa Oil in Hyperoxia-Induced Lung Injury. Arch Bronconeumol. 2013; 49(1): 15-21.

44. Thomson M, Alnaqeeb MA, Bordia T, et al. Effects of aqueous extract of onion on the liver and lung of rats. J Ethnopharmacol. 1998; 61(2): 91-99.

45. Yildiz D. Nicotine, its metabolism and an overview of its biological effects. Toxicon. 2004; 43(6): 619-632.

46. Yildiz D, Ercal N, Armstrong DW. Nicotine enantiomers and oxidative stress. Toxicology. 1998; 130(2-3): 155-165, indexed in Pubmed: 9865482. 